\title{
Pinning down the cosmic ray source mechanism with new IceCube data
}

\author{
Luis A. Anchordoqui, ${ }^{1}$ Haim Goldberg, ${ }^{2}$ Morgan H. Lynch, ${ }^{1}$ \\ Angela V. Olinto, ${ }^{3,4}$ Thomas C. Paul, ${ }^{1,2}$ and Thomas J. Weiler ${ }^{5}$ \\ ${ }^{1}$ Department of Physics, University of Wisconsin-Milwaukee, Milwaukee, WI 53201, USA \\ ${ }^{2}$ Department of Physics, Northeastern University, Boston, MA 02115, USA \\ ${ }^{3}$ Department of Astronomy and Astrophysics, Enrico Fermi Institute, University of Chicago, Chicago, Il 60637, USA \\ ${ }^{4}$ Kavli Institute for Cosmological Physics, University of Chicago, Chicago, Il 60637, USA \\ ${ }^{5}$ Department of Physics and Astronomy, Vanderbilt University, Nashville TN 37235, USA
}

(Dated: June 20, 2013)

\begin{abstract}
Very recently the IceCube Collaboration has reported an observation of 28 neutrino candidates with energies between $50 \mathrm{TeV}$ and $2 \mathrm{PeV}$, constituting a $4.1 \sigma$ excess compared to the atmospheric background. In this article we investigate the compatibility between the data and a hypothesized unbroken power-law neutrino spectrum for various values of spectral index $\Gamma \geq 2$. We show that $\Gamma \sim 2.3$ is consistent at the $\sim 1.5 \sigma$ level with the observed events up to $2 \mathrm{PeV}$ and to the null observation of events at higher energies. We then assume that the sources of this unbroken spectrum are Galactic, and deduce (i) an energy-transfer fraction from parent protons to pions (finding $\epsilon_{\pi^{ \pm}}$ and $\epsilon_{\pi}$ ), and (ii) a way of discriminating among models which have been put forth to explain the "knee" and "ankle" features of the cosmic ray spectrum. Future IceCube data will test the unbroken power law hypothesis and provide a multi-messenger approach to explaining features of the cosmic ray spectrum, including the transition from Galactic to extragalactic dominance.
\end{abstract}

PACS numbers: 98.70.Sa, 95.85.Ry

\section{INTRODUCTION}

In April 2013 the IceCube Collaboration published an observation of two $\sim 1 \mathrm{PeV}$ neutrinos, with a p-value $2.8 \sigma$ beyond the hypothesis that these events were atmospherically generated [1. These two candidates were found in a search for events with a significant energy deposition as expected for cosmogenic neutrinos [2]. Results of a new search technique designed to extend the range of energy sensitivity were reported in [3. In the new search protocol, selected events were required to start inside an inner fiducial volume of the detector. The fact that neutrinos are produced in high energy cosmic ray events means that atmospheric neutrinos of sufficiently high energy and sufficiently small zenith angle will be accompanied by a muon of the same event and therefore excluded from the sample as entering muons. The veto has been derived explicitly in [4] only for muon neutrinos accompanied by the muon from the same decay. This technique is particularly effective for energies $E_{\nu}>100 \mathrm{TeV}$ and zenith angles less than $60^{\circ}$ or $70^{\circ}$, where the boost is sufficient to ensure that the shower muons and neutrinos follow nearly identical trajectories.

The new analysis revealed an additional 26 neutrino candidates depositing "electromagnetic equivalent energies" ranging from about $30 \mathrm{TeV}$ up to $250 \mathrm{TeV}$. Seven of the events show visible evidence of a muon track, and the remainder are consistent with cascade events. The quoted background estimate from atmospheric neutrinos is $10.6_{-3.6}^{+5.0}$. Taken together, the total sample of 28 events departs from the atmospherically-generated neutrino hypothesis by $4.1 \sigma$.

Interpreting these results in terms of popular astrophysical models appears to be challenging. First of all, if the neutrino flux is indeed a Fermi-shock flux falling as an unbroken $E_{\nu}^{-2}$ power-law, one would expect about 8-9 events above $1 \mathrm{PeV}$, which thus far are not observed. This null result at high-energy may be indicative of a cutoff in the spectrum at $1.6_{-0.4}^{+1.5} \mathrm{PeV}[\underline{3}$. On the other hand, the null result may indicate a steeper but still unbroken $E_{\nu}^{-\Gamma}$ spectrum, with $\Gamma>2$. An interesting issue for the future is whether the data offers directional information about the sources. Neither auto-correlation studies of the data, nor cross-correlation studies of the data with candidate source types, have yet been reported. The IceCube angular resolution for shower events is poor, $15^{\circ}$, so firm conclusions are elusive at present.

In this work we investigate the compatibility between the IceCube observations and the hypothesis of an unbroken power-law spectrum arising from optically thin Galactic neutrino sources. The layout of the paper is as follows. We begin in Sec II by studying which are the possible source spectral indices that are consistent with the data reported thus far. We next assume that the neutrino sources are Galactic in origin, and turn our attention to two interesting consequences of the Galactic power-law hypothesis. The first is the implication for spectral features observed in the cosmic ray (CR) energy spectrum. The second is an implication for the average efficiency of the energy transfer from protons to the charged pions which decay to yield the cosmic neutrino flux. Namely, assuming the neutrinos are indeed of Galactic origin, in Sec. III we explore what IceCube data may tell us about competing theories describing the region of the (baryonic) cosmic ray flux transition from Galactic to extragalactic dominance. After that, in Sec. IV we deduce the energy transfer fraction from the parent protons to the pions which ultimately produce the observed neutri- 
nos, demonstrating that $p p$ collisions are more likely to produce the neutrino flux than are $p \gamma$ collisions. As we based our arguments on the hypothesis that the IceCube neutrino excess originates from optically thin Galactic sources, it is natural to consider evidence which may corroborate or refute this hypothesis. Very recently it has been argued that existing photon bounds could call into question the possibility of a (predominantly) Galactic origin for the IceCube neutrino excess [5]. In Sec. V we revisit the subject, considering the impact of photon bounds as well as other factors relevant to the veracity of our model. Finally in Sec. VI we make a few observations on the consequences of the overall picture discussed herein.

In a complementary fashion to this paper, other authors have recently explored potential extragalactic neutrino sources [6] and new massive particle physics [7] as explanations of the IceCube data. For a recent review see $[8]$.

\section{SPECTRAL SHAPE}

Herein we hypothesize that the cosmic neutrino flux per flavor, averaged over all three flavors, follows an unbroken power law of the form

$$
\frac{d F_{\nu}}{d \Omega d A d t d E_{\nu}}=\Phi_{0}\left(\frac{E_{\nu}}{1 \mathrm{GeV}}\right)^{-\Gamma}
$$

for a factor of several or more above the highest energies so far observed. We ask "What value(s) of the spectral index $\Gamma$ (if any) are consistent with the recent IceCube observations?" We partition observations into three bins: (i) 26 events from $50 \mathrm{TeV}$ to $1 \mathrm{PeV}$, which includes the $\sim 10$ atmospheric background events; (ii) 2 events from $1 \mathrm{PeV}$ to $2 \mathrm{PeV}$; (iii) zero events above $2 \mathrm{PeV}$, say from $2 \mathrm{PeV}$ to $10 \mathrm{PeV}$, with a background of zero events.

For various spectral indices from 2.0 to 2.8 , we fit the neutrino flux to each of these three bins, by integrating over the energy span of the bin. A key point is that we employ IceCube's energy-dependent, flavordependent exposure functions for the 662 days of observation time reported thus far. The IceCube exposures are shown in Fig. 1 .

Our results are summarized in Table II. Column two (three) shows the fitted flux normalization $\Phi_{0}$ for the first (second) bin. The null, third bin requires more explanation: According to the statistics of small numbers 9 , any flux yielding more than 1.29 (2.44) events in the null $2-10 \mathrm{PeV}$ range of bin three, is excluded at $68 \% \mathrm{CL}(90 \%$ CL). Accordingly, columns four and five show the maximum flux normalizations allowed by the null bin three, at the $68 \%$ and $90 \%$ CL's.

Under the assumption of a single power-law across the three energy bins, consistency requires that the maximum flux normalization determined by bin three must exceed the flux normalizations from bins one and two.

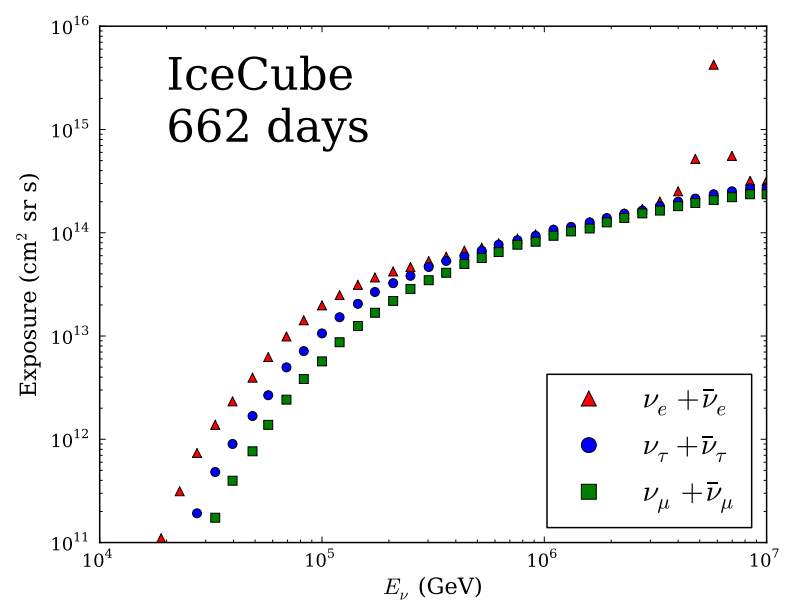

FIG. 1: IceCube exposure for 662 days of data collection. The sharp-peaked structure for $\bar{\nu}_{e}$ at $6.3 \mathrm{PeV}$ is due to the Glashow resonance.

TABLE I: Flavor-averaged normalization $\Phi_{0}$ for the "low energy" $(E<1 \mathrm{PeV})$ and "high energy" (1-2 PeV) bins, and normalization upper limits for the "null" bin $(2-10 \mathrm{PeV})$ at $68 \% \mathrm{CL}\left(\Phi_{68}^{\max }\right)$ and $90 \% \mathrm{CL}\left(\Phi_{90}^{\max }\right)$ in units of $\left(\mathrm{GeV} \cdot \mathrm{cm}^{2} \cdot \mathrm{s} \cdot\right.$ $\mathrm{sr})^{-1}$, for various spectral indices, $\Gamma$.

\begin{tabular}{|c|c|c|c|c|}
\hline \hline$\Gamma$ & $\Phi_{0}^{E_{\nu}<1 \mathrm{PeV}}$ & $\Phi_{0}^{1 \mathrm{PeV}<E_{\nu}<2 \mathrm{PeV}}$ & $\Phi_{68}^{\max }$ & $\Phi_{90}^{\max }$ \\
\hline 2.0 & $1.66 \times 10^{-8}$ & $9.50 \times 10^{-9}$ & $3.94 \times 10^{-9}$ & $7.44 \times 10^{-9}$ \\
\hline 2.1 & $5.70 \times 10^{-8}$ & $3.91 \times 10^{-8}$ & $1.84 \times 10^{-8}$ & $3.49 \times 10^{-8}$ \\
\hline 2.2 & $1.95 \times 10^{-7}$ & $1.61 \times 10^{-7}$ & $8.62 \times 10^{-8}$ & $1.63 \times 10^{-7}$ \\
\hline 2.3 & $6.63 \times 10^{-7}$ & $6.62 \times 10^{-7}$ & $4.02 \times 10^{-7}$ & $7.61 \times 10^{-7}$ \\
\hline 2.4 & $2.24 \times 10^{-6}$ & $2.72 \times 10^{-6}$ & $1.88 \times 10^{-6}$ & $3.55 \times 10^{-6}$ \\
\hline 2.5 & $7.54 \times 10^{-6}$ & $1.12 \times 10^{-5}$ & $8.73 \times 10^{-6}$ & $1.65 \times 10^{-5}$ \\
\hline 2.6 & $2.52 \times 10^{-5}$ & $4.59 \times 10^{-5}$ & $4.06 \times 10^{-5}$ & $7.68 \times 10^{-5}$ \\
\hline 2.7 & $8.39 \times 10^{-5}$ & $1.88 \times 10^{-4}$ & $1.88 \times 10^{-4}$ & $3.56 \times 10^{-4}$ \\
\hline 2.8 & $2.78 \times 10^{-4}$ & $7.71 \times 10^{-4}$ & $8.73 \times 10^{-4}$ & $1.65 \times 10^{-3}$ \\
\hline \hline
\end{tabular}

Moreover, the fitted normalizations from bins one and two should be the same, or nearly so. In terms of the Table columns, if flux numbers from columns two or three exceed the maximums of columns 4 and 5 , then the fit is ruled out at $68 \%$ and $90 \%$ CL. Table I reveals that spectral indices shallower than 2.3 are inconsistent with the data at $90 \%$ CL or more, while indices shallower than 2.7 are inconsistent at $68 \%$ CL. Only for $\Gamma=2.3$ are the normalizations from bins one $\left(E_{\nu}<1 \mathrm{PeV}\right)$ and two $\left(1 \mathrm{PeV}<E_{\nu}<2 \mathrm{PeV}\right)$ quite consistent with each other, and therefore with an unbroken power law. The overall consistency of the $\Gamma=2.3$ power law across all three bins is at roughly the $1.5 \sigma$ level. We therefore choose $\Gamma=2.3$ as our reference value for the unbroken power law hypothesis. Taking into account the errors on the 
background in the first bin reported by the IceCube collaboration, we find $\Gamma=2.3 \pm 0.2$ (with normalization given in the third column of Table I). A recent analysis performed prior to the announcement of the 26 events below $1 \mathrm{PeV}$ is consistent with our finding [10].

\section{TRANSITION FROM GALACTIC TO EXTRAGALACTIC SOURCES}

Above about $10 \mathrm{GeV}$, the $\mathrm{CR}$ energy spectrum is observed to fall roughly as a power law; the flux decreases nearly three orders of magnitude per energy decade until eventually suffering a strong suppression near $60 \mathrm{EeV}$ [1]. Close examination reveals several other spectral features. A steepening of the spectrum from $J(E) \propto E^{-2.67 \pm 0.07}$ to $E^{-3.07 \pm 0.11}$ has been dubbed the "knee" occurring at $E_{\text {knee }} \approx 3 \mathrm{PeV}[12$. A less prominent "second knee", corresponding to a further softening $J(E) \propto E^{-3.52 \pm 0.19}$ appears above $0.3 \mathrm{EeV}$ [13]. At $E_{\text {ankle }} \approx 3 \mathrm{EeV}$ a pronounced hardening of the spectrum becomes evident, generating the so-called "ankle" feature [14.

The small variations of the spectral index can be interpreted either as a transition between CR populations or as an imprint of $\mathrm{CR}$ propagation effects. One model posits that extragalactic protons dominate the CR composition at and above the second knee, and that the ankle feature is carved into the spectrum as a result of $e^{+} e^{-}$pair production when $\mathrm{CR}$ protons interact with the cosmic microwave background (CMB) photons [15]. This model is often referred to as the "dip" model. In contrast, a second model proposes that the ankle feature represents a crossover of the two fluxes, Galactic and extragalactic, with different spectral indices, signifying a transition from heavy nuclei of Galactic origin to proton dominance of the extra-galactic spectrum [16].

If the ankle marks the Galactic to extra-galactic CR transition, then Galactic sources must be able to accelerate nuclei up to about 1-3 EeV [17. Assuming that the highest energies attainable from a Galactic sources scale as $E=Z E_{p}$, then protons should be accelerated to only about $1 / 26$ of the energy of the ankle, or $E_{p} \sim 120 \mathrm{PeV}$. Proton interactions with either photons or other (low energy) protons at the acceleration sites ultimately give rise to neutrinos which carry on average $\sim 1 / 16$ of the initial proton energy [18]. The neutrino spectrum has the same spectral index as the hard protons at the source. Thus, if the proton spectrum follows an unbroken power law up to a maximum energy of $\sim 120 \mathrm{PeV}$, neutrinos produced by proton interactions in Galactic sources should exhibit an unbroken power law which extends to roughly 8-10 PeV, but not beyond. On the other hand, the dip model places the Galactic to extragalactic transition in the region of the second knee, $E \sim 500 \mathrm{PeV}$. This implies, by our previous arguments, a maximum Galactic proton energy 26 times smaller, $E_{p} \sim 20 \mathrm{PeV}$, and a maximum neutrino energy 16 times smaller again, $E_{\nu} \sim 1 \mathrm{PeV}$.
So far, no events from 2-10 PeV have been observed. As we have shown above, this null result presents only a $1.5 \sigma$ downward fluctuation for an unbroken power law with spectral index $\Gamma=2.3$. So the jury is out, awaiting further IceCube data for the $2-10 \mathrm{PeV}$ region. If an unbroken neutrino power law is ultimately confirmed all the way to $\sim 10 \mathrm{PeV}$, this would naturally favor the ankle transition model, as some fine tuning would then be required for the dip model. On the other hand, if future observations continue the null view of the $2-10 \mathrm{PeV}$ region, then the dip model becomes favored.

It could, of course, be the case that an extragalactic component contributes beyond $\sim 2 \mathrm{PeV}$, although extraordinary fine tuning would be required for the spectral indices to be the same above and below the galactic to extragalactic transition. If the Galactic sources begin to reach the end of their acceleration potential, one would expect a break in the index, characterized by a steepening of the spectrum. In contrast, if an extragalactic contribution with a shallow spectrum induces the break, a hardening of the spectrum is expected above the transition. Ultimately, IceCube will achieve the capacity to isolate the sources or source populations of the highest energy neutrinos, delivering the final verdict.

Given that the CR spectrum exhibits breaks at the knee and second knee, we should ask whether it is plausible for the proton injection spectrum to be characterized by a single index over the energy range of interest. If neutrinos are produced at the same sites as the CRs, then there are two categories of models which may explain these breaks; the knee may signify the acceleration endpoint of one of two types of sources [19], or the knee may result from magnetic-dependent leakage of particles from the Galaxy 20. If the latter is correct then the injected proton spectrum, and hence neutrino spectrum, should follow an unbroken power law over the energy ranges under discussion here. Thus, the shape of the neutrino spectrum arriving from the Galactic disk will also help to discriminate among these competing knee models. It may also be the case that neutrino production during propagation is relevant. Qualitatively speaking, if this effect is non-negligible but not dominant, one would expect a hardening of the neutrino spectrum with energy. In contrast, if neutrinos are predominantly generated during propagation, the spectrum should soften with energy [21.

To quantify the spectral features characteristic of these two models we adopt the "leaky box" picture, in which CRs propagate freely in the Galaxy, contained by the magnetic field but with some probability to escape which is constant in time. The local energy density is given by

$$
n_{\mathrm{CR}}(E) \equiv \frac{4 \pi}{c} J(E) \approx Q(E) \tau(E / Z)
$$

where $Q(E) \propto E^{-\alpha}$ is the generation rate of primary CRs and $\tau(E / Z) \propto E^{-\delta}$ is the rigidity-dependent confinement time 22]. Fits to the energy dependence of secondary to primary ratios yield $\delta=0.6$ [23]. For a 
source index $\alpha \simeq 2.07$, which is close to the prediction of Fermi shock acceleration, inclusion of propagation effects reproduces the observed spectrum. However, $\delta=0.6$ results in an excessively large anisotropy which is inconsistent with observations 24. Consistency with anisotropy can be achieved by adopting a Kolmogorov index, $\delta=1 / 3$ [19, 20]. The apparent conflict with the secondary to primary composition analyses can be alleviated through small variations of the energy dependence of the spallation cross sections, or variation in the matter distribution in the Galaxy [19]. This hypothesis implies a steeper source spectrum, $\alpha \simeq 2.34$, which agrees remarkably well with the fit of an unbroken power law to IceCube data, as discussed herein.

We consider a model in which cosmic ray leakage is dominated by Kolmogorov diffusion, $\tau \propto(E / Z)^{-1 / 3}$, for $E<Z E_{\text {knee, }}$ with increasing leakage due to decreasing trapping efficiency with rising energy, $\tau \propto(E / Z)^{-1}$ for $E \gg Z E_{\text {knee }}[20$. The knee is etched into the spectrum by a transition from diffusion to drift motion, while the second knee results from a subsequent transition to quasirectilinear motion. Each CR nucleus is affected by drifts at $E \simeq Z E_{\text {knee, }}$, resulting in a progressive steepening of the CR spectrum. Since the lighter component are strongly suppressed above $0.1 \mathrm{EeV}$ we are left with an iron dominated spectrum which progressively steepens until the overall spectrum becomes $J(E) \propto E^{-2.67-2 / 3}$, in agreement with observation of the second knee [13].

It is helpful to envision the CR engines as machines where protons are accelerated and (possibly) permanently confined by the magnetic fields of the acceleration region. The production of neutrons and pions and subsequent decay produces neutrinos, $\gamma$-rays, and CRs. If the neutrino-emitting source also produces high energy CRs, then pion production must be the principal agent for the high energy cutoff on the proton spectrum. Conversely, since the protons must undergo sufficient acceleration, inelastic pion production needs to be small below the cutoff energy; consequently, the plasma must be optically thin. Since the interaction time for protons is greatly increased over that of neutrons due to magnetic confinement, the neutrons escape before interacting, and on decay give rise to the observed CR flux. The foregoing can be summarized as three conditions on the characteristic nucleon interaction time scale $\tau_{\text {int }}$; the neutron decay lifetime $\tau_{n}$; the characteristic cycle time of confinement $\tau_{\text {cycle }}$; and the total proton confinement time $\tau_{\text {conf }}:(i) \tau_{\text {int }} \gg \tau_{\text {cycle }}$; (ii) $\tau_{n}>\tau_{\text {cycle }} ;$ (iii) $\tau_{\text {int }} \ll \tau_{\text {conf }}$. The first condition ensures that the protons attain sufficient energy. Conditions $(i)$ and $(i i)$ allow the neutrons to escape the source before decaying. Condition (iii) permits sufficient interaction to produce neutrons and neutrinos. These three conditions together define an optically thin source. In what follows we assume these three conditions hold for some neutrino-emitting sources in the Galaxy.

As an illustration, we mention astrophysical environments where the conditions discussed above could hold. The Galactic Center, for instance, has been proposed as a source candidate 25, 26. These conditions can also apply in the jets of powerful microquasars where protons can be efficiently accelerated beyond the knee feature. Neutrino production in $p \gamma$ [27] and $p p$ [28] collisions has been suggested as a possible source of neutrinos. Attaining the maximum observed neutrino energies for such scenarios may require fine tuning and pushing parameters to their extrema, to which one may object. The assumption that sources with the requisite properties do exist is, however, consistent with a very general estimate of Galactic cosmic-ray power required to match the observed spectrum up to about the second knee, as well as a rough estimate of the ratio of heavy nuclei to protons as measured by KASCADE near the end of the presumed Galactic spectrum. We elaborate upon these points in the next section. Whatever point of view one may find most convincing, however, we should rely on future experimental results rather than "naturalness" to settle the question.

\section{POWER FOR GALACTIC COSMIC RAYS}

Next we turn to the question of what the Galactic power-law model developed above would imply regarding the average efficiency of transferring proton energy to charged pions. Assume that the source spectral index of CRs in the range $0.1-100 \mathrm{PeV}$ is $\Gamma$ from here on. Then, following [18, we define the two constants

$$
C_{\mathrm{CR}}^{p}(\Gamma) \equiv \frac{d F_{\mathrm{CR}}^{p}}{d E d A d t} E^{\Gamma}, \text { and } C_{\nu}(\Gamma) \equiv \frac{d F_{\nu}}{d E d A d t} E^{\Gamma},
$$

where $C_{\nu}=4 \pi \Phi_{0}^{\text {total }} \mathrm{GeV}^{\Gamma}$ and $\Phi_{0}^{\text {total }}=3 \Phi_{0}$, given our assumption of flavor equilibration. In conventional notation, we next define $\epsilon_{\pi^{ \pm}}$to be the ratio of CR power (energy/time) emitted in charged pions to that in the parent nucleons. We also need $\epsilon_{\nu}$, defined as the fractional energy in neutrinos per single charged pion decay. If the pion decay chain is complete $\left(\pi^{ \pm} \rightarrow e+\nu_{e}+\nu_{\mu}+\bar{\nu}_{\mu}\right)$, then $\epsilon_{\nu} \simeq 3 / 4$, whereas if the pion decay chain is terminated in the source region by energy loss of the relatively long-lived muon, then $\epsilon_{\nu} \simeq 1 / 4$. Comparing the energy produced in charged pions at the source to the neutrino energy detected at Earth, one gets the energy conservation relation

$$
\epsilon_{\nu} \epsilon_{\pi^{ \pm}} \int_{E_{1}}^{E_{2}} \frac{d F_{\mathrm{CR}}^{p}}{d E d A d t} E d E=\int_{E_{\nu 1}}^{E_{\nu 2}} \frac{d F_{\nu}}{d E_{\nu} d A d t} E_{\nu} d E_{\nu},
$$

where $E_{\nu 1}=\frac{E_{1}}{16}$, and $E_{\nu 2}=\frac{E_{2}}{16}$; these integrals may be done analytically to yield (for $\Gamma \neq 2$ )

$$
\epsilon_{\nu} \epsilon_{\pi^{ \pm}} C_{\mathrm{CR}}^{p} \frac{E_{1}^{2-\Gamma}-E_{2}^{2-\Gamma}}{\Gamma-2}=\frac{\left(\frac{E_{1}}{16}\right)^{2-\Gamma}-\left(\frac{E_{2}}{16}\right)^{2-\Gamma}}{\Gamma-2} C_{\nu} .
$$

Then, solving for $\epsilon_{\pi^{ \pm}}$we arrive at

$$
\epsilon_{\pi^{ \pm}}=\left(\frac{1}{16}\right)^{2-\Gamma} \frac{C_{\nu}(\Gamma)}{\epsilon_{\nu} C_{\mathrm{CR}}^{p}(\Gamma)} .
$$


The numerology for $C_{\nu}$ is given in Table 1 . For the favored spectral index $\Gamma=2.3$, we have

$$
C_{\nu}(2.3)=12 \pi \times 6.6 \times 10^{-7} \mathrm{GeV}^{2.3}\left(\mathrm{GeV} \mathrm{s} \mathrm{cm}^{2}\right)^{-1} .
$$

The constant $C_{\mathrm{CR}}^{p}(2.3)$ is related to the injection power of CR protons, $d \epsilon_{\mathrm{CR}}^{p} / d t$, as follows:

$$
\begin{aligned}
\frac{d \epsilon_{\mathrm{CR}}^{p}}{d t}\left[E_{1}, E_{2}\right] & =A \int_{E_{1}}^{E_{2}} \frac{d F_{\mathrm{CR}}^{p}}{d E d A d t} E d E \\
& =A \int_{E_{1}}^{E_{2}}\left(\frac{d F_{\mathrm{CR}}^{p}}{d E d A d t} E^{\Gamma}\right) E^{(1-\Gamma)} d E \\
& =A C_{\mathrm{CR}}^{p} \frac{\left(E_{1}{ }^{(2-\Gamma)}-E_{2}{ }^{(2-\Gamma)}\right)}{\Gamma-2}
\end{aligned}
$$

where $A=4 \pi r^{2}$ is an appropriately weighted surface area for the arriving cosmic-ray or neutrino flux. In [29], $A$ is set equal to $4 \pi R_{\mathrm{G}}^{2} \equiv A_{0}$, where $R_{\mathrm{G}}$ is the Galactic radius, $\approx 10 \mathrm{kpc}$. However, keeping in mind that $\left\langle r^{-2}\right\rangle$ diverges as $\ln \left(R_{\mathrm{G}} / 2 r_{\min }\right)$, with $r_{\text {min }}$ being the distance to the nearest source, $A^{-1}$ can easily be a factor of 2 larger than $A_{0}^{-1}$. Two independent arguments support such an enhancement. The first is to simply note that a local void radius of $0.7 \mathrm{kpc}$ gives $A_{0} / A=2$. The second is to note that the thin-disk approximation breaks down at a small distance $z$ of order of the disk height, leading to a similar guesstimate of integration cutoff and resulting enhancement factor. Inverting (5) and using the fact that $E_{2}{ }^{(2-\Gamma)} \ll E_{1}{ }^{(2-\Gamma)}$, we get the conversion

$$
C_{\mathrm{CR}}^{p}=\frac{d \epsilon_{\mathrm{CR}}^{p}}{d t}\left[E_{1}, E_{2}\right] \frac{(\Gamma-2) E_{1}^{(\Gamma-2)}}{A} .
$$

How, and how well, is $d \epsilon_{\mathrm{CR}}^{p} / d t$ known? The assumption underlying the leaky box model is that the energy density in CRs observed locally is typical of other regions of the Galactic disk. If so, the total power required to maintain the cosmic radiation in equilibrium can be obtained by integrating the generation rate of primary CRs over energy and space. Using (2), we obtain

$$
\frac{d \epsilon_{\mathrm{CR}}}{d t}=\int d^{3} x \int Q(E) d E=V_{G} \frac{4 \pi}{c} \int \frac{J(E)}{\tau(E / Z)} d E,
$$

where $V_{G} \sim 10^{67} \mathrm{~cm}^{3}$ is the Galactic disk volume 30. For $E_{\text {knee }}<E<E_{\text {ankle, }}$, we conservatively assume that the trapping time in the Galaxy scales with energy as $\tau=2 \times 10^{7}\left(E_{\mathrm{GeV}} / Z\right)^{-1 / 3}$ yr 31 . (Note that an evolution into quasirectilinear motion would increase the power allowance.) In this case the power budget required to fill in the spectrum from the knee to the ankle is found to be $d \epsilon_{\mathrm{CR}} / d t \simeq 2 \times 10^{39} \mathrm{erg} / \mathrm{s}$ [31].

We also note that recent data from KASCADEGrande 32 indicate that at $\sim 30 \mathrm{PeV}$ the flux of protons is about an order of magnitude smaller than the allspecies CR flux. Taken at face value, this implies that the fraction of the power budget allocated to nucleons of energy $E_{p}$ which do not escape the Galaxy is about 0.1 of the all-species power. However, light elements possess higher magnetic rigidity and are therefore more likely to escape the Galaxy. From the functional form of $\tau(E / Z)$ above, we estimate the survival probability for protons at $30 \mathrm{PeV}$ to be $46 \%$ of that at $E_{\text {knee. }}$ This leads to a value for the proton fraction of total flux at injection $(\zeta)$ of $\zeta=0.1 / 0.46=0.22$. In our analysis, we will consider a wide range for $\bar{\zeta} \equiv \zeta A_{0} / A$, with $0.22 \lesssim \bar{\zeta} \lesssim 0.44$ seemingly the most realistic range.

Then, we find for $C_{\mathrm{CR}}^{p}$ the particular result

$$
C_{\mathrm{CR}}^{p}(2.3)=\frac{0.3 \times(0.1 \mathrm{PeV})^{0.3} \times 2 \bar{\zeta} \times 10^{39} \mathrm{erg} / \mathrm{s}}{4 \pi(10 \mathrm{kpc})^{2}} .
$$

Finally, inserting Eqs. (4) and (8) into (3), we get

$$
\epsilon_{\pi^{ \pm}}(2.3)=\left(\frac{1}{16}\right)^{-0.3} \frac{C_{\nu}(2.3)}{\epsilon_{\nu} C_{\mathrm{CR}}^{p}(2.3)}=\frac{0.055}{\bar{\zeta} \epsilon_{\nu}}
$$

where in the final expression, we have set $\Gamma$ equal to our favored value of 2.3 33. If neutrinos are produced in $p p$ collisions, one can interpret $\epsilon_{\pi^{ \pm}}$in terms of the efficiency of transferring proton energy to all three pion species, $\epsilon_{\pi}$, by simply scaling $\epsilon_{\pi^{ \pm}}$by $3 / 2$. Alternatively, if neutrinos are produced in $p \gamma$ collisions, we scale $\epsilon_{\pi^{ \pm}}$by 2 34. We show $\epsilon_{\pi}\left(\bar{\zeta}=\zeta A_{0} / A\right)$ for all four cases in Fig. 2,

In $p p$ collisions, hadronic models predict that $f_{\pi} \sim 0.6$ of the "beam" proton energy is channeled into pions [35. Since the value of $\epsilon_{\pi}$ reflects both the inelasticity as well as the fraction of protons which escape the source without producing pions, we expect $\epsilon_{\pi}$ to be smaller than $f_{\pi}$. This turns out to be the case for a complete pion decay chain if $\zeta A_{0} / A>0.19$. Note, however, that the incomplete pion decay chain requires a considerably larger fraction, $\zeta A_{0} / A>0.59$, which pushes the realm of plausibility. For $p \gamma$ interactions, $f_{\pi} \sim 0.28$ [36], thereby excluding the incomplete decay chain hypothesis for this case. On the other hand, the complete decay chain appears to be allowed only for $\zeta A_{0} / A>0.56$.

\section{CONSISTENCY WITH PHOTON LIMITS AND ARRIVAL DIRECTION DISTRIBUTION}

It is interesting to employ existing limits on high energy photons to check the plausibility of our hypothesis that the IceCube excess is of Galactic origin. $\gamma$ rays are produced by $\pi^{0}$ decays at the same optically thin sources where neutrinos are produced by $\pi^{ \pm}$decay. As described in [8], once can predict a differential $\gamma$ ray flux based the best-fit single power law $\nu$ flux discussed in this paper, and compare to measurements. The CASA-MIA $90 \%$ C.L. upper limits on the integral diffuse $\gamma$ ray flux, $I_{\gamma}$ for energy bins,

$$
\frac{E_{\gamma}^{\min }}{\mathrm{GeV}}=3.30 \times 10^{5}, 7.75 \times 10^{5}, 2.450 \times 10^{6},
$$




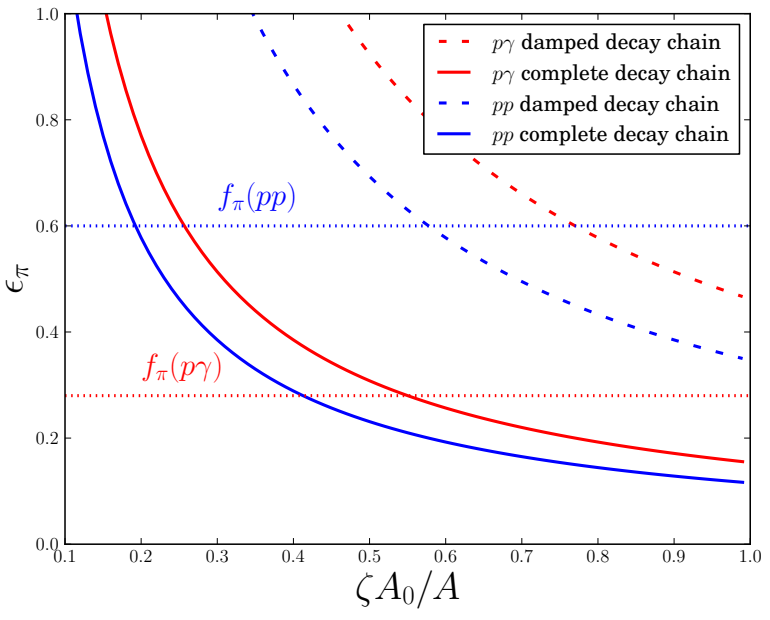

FIG. 2: Total pion energy fractions of parent proton, for favored spectral index $\Gamma=2.3$. The average inelasticity $f_{\pi}$ for $p p$ and $p \gamma$ collisions is also shown for comparison.

are

$$
\begin{aligned}
\frac{I_{\gamma}}{\mathrm{cm}^{-2} \mathrm{~s}^{-1} \mathrm{sr}^{-1}}< & 1.0 \times 10^{-13}, 2.6 \times 10^{-14}, \\
& 2.1 \times 10^{-15},
\end{aligned}
$$

respectively [37. Under the simplifying assumption that there is no photon absorption, the integral photon fluxes we predict based on our single power law hypothesis (in units of photons $\mathrm{cm}^{-2} \mathrm{~s}^{-1} \mathrm{sr}^{-1}$ ), above the energies specified in 100$)$, are

$$
\begin{aligned}
\int_{E_{\gamma}^{\min }} \frac{d F_{\gamma}}{d \Omega d A d t d E_{\gamma}} d E_{\gamma}= & 4.2 \times 10^{-14}, 1.4 \times 10^{-14}, \\
& 3.1 \times 10^{-15} .
\end{aligned}
$$

For the first two energy bins, the predicted fluxes are below the $90 \%$ C.L. measurements of CASA-MIA, while the last bin slightly exceeds the $90 \%$ C.L. bound. This does not, however, imply that the Galactic origin hypothesis is ruled out at $90 \%$ C.L. First of all, one must keep in mind that sources which are optically thin up to $E_{\gamma} \sim 100 \mathrm{TeV}$ may no be optically thin at higher energies, suggesting that the importance of photon bounds in establishing the origin of the IceCube excess should be considered with some caution [38. Even if we ignore this caveat, we still do not know the maximum neutrino energy reached at acceleration sites, so the maximum photon energy is likewise unknown. In addition, absorption becomes important in the energy regime covered by the last bin, as mean free path of $\mathrm{PeV}$ photons in the $\mathrm{CMB}$ is about 10 kpc.

Note that $R_{\mathrm{G}} \sim 10 \mathrm{kpc}$, leading to an interesting signature: Photons coming from "our half" of the Galaxy will be largely unattenuated, while those from the farther half will be significantly attenuated. Since both photons and neutrinos point back to the sources, coordinated comparisons of neutrino and photon data will facilitate a completely new exploration of the highestenergy Galactic sources. As described in [8], taking into account absorption of the photon flux for $E_{\gamma}^{\min }>1 \mathrm{PeV}$ leads to about a $12 \%$ reduction in the predicted photon flux. Furthermore, varying the photon maximum energy cutoff of Eq. 12 to,

$$
\frac{E_{\gamma}^{\max }}{\mathrm{PeV}}=6,7,8,
$$

we obtain

$$
\begin{aligned}
\int_{E_{\gamma}^{\min }}^{E_{\gamma}^{\max }} \frac{d F_{\gamma}}{d \Omega d A d t d E_{\gamma}} d E_{\gamma}= & 2.1 \times 10^{-15}, 2.3 \times 10^{-15} \\
& 2.4 \times 10^{-15}
\end{aligned}
$$

From the discussion above, we can see there are several ways to comply with the CASA-MIA bound. For instance, $E_{\gamma}^{\max }=6 \mathrm{PeV}$ is already consistent with the measured bound, even without absorption. For higher energies, absorption provides enough reduction of the photon flux to retain consistency with measurements. It is also worth noting that the comparison discussed here is based on experimental bounds on the all-sky $\gamma$ ray flux. A more rigorous comparison would involve measurements on the diffuse $\gamma$ fay flux within about $15^{\circ}$ of the Galactic plane. The CASA-MIA Collaboration has in fact studied $\gamma$ ray emission from the direction of the Galactic plane, reporting the flux limits as a fraction of the CR flux [39] rather than an integral bound. Comparing the relative fractions from the all-sky analysis to the Galactic plane analysis indicates that constraining the observation to the Galactic plane region does indeed lead to tighter constraints; the first two energy bins discussed above are roughly saturated at the $90 \%$ C.L. while the bound for the highest energy bin remains roughly the same. Only the IceCube collaboration has thus far reported constraints between 1 and $10 \mathrm{PeV}$. Bounds from the IceCube 40 string configuration [40, are not restrictive enough to challenge the Galactic origin hypothesis. However, within 5 years of data taking with the complete IceCube configuration of 86 strings, enough statistics will be gathered to elucidate the $\nu-\gamma$ ray connections.

Finally we comment on the consistency between the arrival direction distribution of the IceCube excess and the hypothesis that the sources are nearby. Fourteen of the 26 reported neutrino events arrive from within about $15^{\circ}$ of the Galactic plane, including one of the two highest energy events, which coincides with the Galactic center (within errors). The highest energy event is outside of this angular window, but (as noted in [5]) does correspond with a possible hotspot in the IceCube photon search [40. This could reflect emission of neutrinos and $\gamma$ rays from a common, nearby source, as $\gamma$ rays do not survive propagation further than $\sim 10 \mathrm{kpc}$. The recently discovered large reservoir of ionized gas extending over a large region around the Milky Way [41] could provide the target material required for neutrino production outside the Galactic disk in models in which proton diffusion 
extends to the Galactiuc halo [42. However, given the current statistics and the insufficient understanding of the atmospheric (in particular the prompt neutrino [43]) background, the arrival direction distribution neither favors nor disfavors a Galactic origin [5, 25]. More data are required to settle the issue.

\section{CONCLUSIONS}

Summarizing, we embrace this joyous moment that appears to be the dawn of neutrino astronomy, by investigating the hypothesis of a single power-law Galactic neutrino flux, and investigating several further consequences of the hypothesis. Implicit in our phenomenological analysis is the assumption that there exist Galactic cosmic ray sources which are both optically thin and capable of generating protons with energies well beyond the knee feature, and neutrinos with energies around $E_{\nu}=E_{p} / 16$. As discussed above, this assumption may stress acceleration models, but is not excluded by current cosmic ray observations. Combining the assumption that sources with these requisite conditions exist with the hypothesis that the observed neutrino spectrum can be characterized by a single power law leads to three interesting ramifications.

We find that a spectral index of $\sim 2.3$ is consistent with the data over the range $50 \mathrm{TeV}-10 \mathrm{PeV}$, at $1.5 \sigma$. A shallower spectrum overproduces events in the null region above $\sim 2 \mathrm{PeV}$, while a steeper spectrum fails to match the event rate below a $\mathrm{PeV}$ to that at $1-2 \mathrm{PeV}$. The first ramification is that we identified a discriminator between the "dip model" for the Galactic to extragalactic crossover, and the "ankle model." The discriminator is the termination energy of the neutrino spectrum. If it is $1-2 \mathrm{PeV}$, then the "dip model" is favored; if it is 8$10 \mathrm{PeV}$, then the "ankle model" is favored. Secondly, we identified a means of discriminating between competing models for explaining the knee feature. If the knee results from an overlay of spectra for two types of sources, one of which is reaching its acceleration endpoint, we expect to see a break in the neutrino spectrum around $190 \mathrm{TeV}$. If the knee is a consequence of rigidity dependent leakage from the Galaxy, we expect no such break in the neutrino spectrum. The third ramification is that although the resulting energy fraction transferred from parent proton to daughter pions is only 2 to 3 times below the Waxman-Bahcall (WB) bound 44, the neutrino flux beyond $1 \mathrm{PeV}$ requires a steep spectrum $\propto E_{\nu}^{-2.3}$. This has the unfortunate consequence of requiring 1 order of magnitude more years, or 1 order of magnitude larger detector volume, to produce the same event numbers hoped for from saturation of the original WB bound beyond $1 \mathrm{PeV}$.

Thus far the IceCube excess is consistent with a Galactic origin, so we have included all data in our analysis. In the future, however, the data may well show evidence of extragalactic sources. In this case the analysis presented here can be repeated with cuts to exclude extragalactic "contamination," e.g., by requiring events to arrive from within $15^{\circ}$ or so of the Galactic plane.

\section{Acknowledgments}

We thank Francis Halzen, Ruoyu Liu, and Soeb Razzaque for discussion. This work was supported by the US NSF grant numbers: CAREER PHY1053663 (LAA); PHY-0757959 (HG); PHY-1205854 (TCP); PHY-1068696, PHY-1125897 (AVO); US DoE grant DEFG05-85ER40226 (TJW); NASA 11-APRA-0058 (LAA, TCP), 11-APRA-0066 (AVO); and UWM Physics 2013 Summer Research Award (MHL).
[1] M. G. Aartsen et al. [IceCube Collaboration], Phys. Rev. Lett. 111, 021103 (2013) arXiv:1304.5356.

[2] V. S. Berezinsky and G. T. Zatsepin, Phys. Lett. B 28, 423 (1969).

[3] M. G. Aartsen et al. [IceCube Collaboration], Science 342, no. 6161, 1242856 (2013) arXiv:1311.5238 [astroph.HE]]; F. Halzen, arXiv:1308.3171.

[4] S. Schonert, T. K. Gaisser, E. Resconi and O. Schulz, Phys. Rev. D 79, 043009 (2009) arXiv:0812.4308.

[5] M. Ahlers and K. Murase, arXiv:1309.4077 [astroph.HE].

[6] I. Cholis and D. Hooper, JCAP 06, 030 (2013) arXiv:1211.1974; M. D. Kistler, T. Stanev and H. Yuksel, arXiv:1301.1703, O. E. Kalashev, A. Kusenko and W. Essey, Phys. Rev. Lett. 111, 041103 (2013) arXiv:1303.0300; F. W. Stecker, Phys. Rev. D 88, 047301 (2013) arXiv:1305.7404; K. Murase and K. Ioka, arXiv:1306.2274 K. Murase, M. Ahlers and B. C. Lacki, arXiv:1306.3417.
[7] B. Feldstein, A. Kusenko, S. Matsumoto and T. T. Yanagida, arXiv:1303.7320 V. Barger and W. -Y. Keung, arXiv:1305.6907.

[8] L. A. Anchordoqui, V. Barger, I. Cholis, H. Goldberg, D. Hooper, A. Kusenko, J. G. Learned, D. Marfatia, S. Pakvasa, T. C. Paul, and T. J. Weiler, JHEAp 1, 1 (2014) arXiv:1312.6587 [astro-ph.HE]].

[9] G. J. Feldman and R. D. Cousins, Phys. Rev. D 57, 3873 (1998) arXiv:physics/9711021.

[10] R. Laha, J. F. Beacom, B. Dasgupta, S. Horiuchi and K. Murase, Phys. Rev. D 88, 043009 (2013) arXiv:1306.2309.

[11] R. Abbasi et al. [HiRes Collaboration], Phys. Rev. Lett. 100, 101101 (2008) arXiv:astro-ph/0703099; J. Abraham et al. [Pierre Auger Collaboration], Phys. Rev. Lett. 101, 061101 (2008) arXiv:0806.4302.

[12] J. R. Hörandel, Astropart. Phys. 19, 193 (2003) arXiv:astro-ph/0210453 ; P. Blasi and E. Amato, JCAP 1201, 010 (2012) arXiv:1105.4521. 
[13] T. Abu-Zayyad et al., Astrophys. J. 557, 686 (2001) arXiv:astro-ph/0010652.

[14] For a review see e.g., J. Blümer, R. Engel and J. R. Hörandel, Prog. Part. Nucl. Phys. 63, 293 (2009) arXiv:0904.0725.

[15] M. Hillas, Phys. Lett. A 24, 677 (1967); V. Berezinsky, A. Z. Gazizov and S. I. Grigorieva, Phys. Rev. D 74, 043005 (2006) arXiv:hep-ph/0204357.

[16] J. Linsley, 8th ICRC (Jaipur, India, 1963); C. T. Hill and D. N. Schramm, Phys. Rev. D 31, 564 (1985).

[17] Sources with sufficient energetics are discussed in, L. A. Anchordoqui, H. Goldberg, F. Halzen and T. J. Weiler, Phys. Lett. B 593, 42 (2004) arXiv:astro-ph/0311002; L. A. Anchordoqui, J. F. Beacom, H. Goldberg, S. Palomares-Ruiz and T. J. Weiler, Phys. Rev. D 75, 063001 (2007) arXiv:astro-ph/0611581; P. Blasi, R. I. Epstein and A. V. Olinto, Astrophys. J. 533, L123 (2000) arXiv:astro-ph/9912240; K. Fang, K. Kotera and A. V. Olinto, JCAP 1303, 010 (2013) arXiv:1302.4482; P. L. Biermann, G. A. Medina-Tanco, R. Engel and G. Pugliese, Astrophys. J. 604, L29 (2004) arXiv:astro$\mathrm{ph} / 0401150$.

[18] M. Ahlers, L. A. Anchordoqui, H. Goldberg, F. Halzen, A. Ringwald and T. J. Weiler, Phys. Rev. D 72, 023001 (2005) arXiv:astro-ph/0503229.

[19] P. L. Biermann Astron. Astrophys. 271, 649 (1993); P. L. Biermann, T. K. Gaisser and T. Stanev, Phys. Rev. D 51, 3450 (1995) arXiv:astro-ph/9501001. A. M. Hillas, J. Phys. G 31, R95 (2005).

[20] S. I. Syrovatskii, Comment. Astrophys. Space Phys. 3, 155 (1971); V. S. Ptuskin, S. I. Rogovaya, V. N. Zirakashvili, L. G. Chuvilgin, G. B. Khristiansen, E. G. Klepach, G. V. Kulikov, Astron. Astrophys. 268, 726 (1993); J. Candia, S. Mollerach and E. Roulet, JCAP 0305, 003 (2003) arXiv:astro-ph/0302082.

[21] J. Candia and E. Roulet, JCAP 0309, 005 (2003) astroph/0306632 ; J. Candia, JCAP 0511, 002 (2005) astroph/0505346.

[22] T. K. Gaisser, "Cosmic rays and particle physics," (Cambridge, UK: Univ. Press, 1990).

[23] M. Gupta and W. R. Webber, Astrophys. J. 340, 1124 (1989); S. P. Swordy, J. L'Heureux, P. Meyer and D. Muller, Astrophys. J. 403, 658 (1993).

[24] P. Blasi and E. Amato, JCAP 1201, 011 (2012) arXiv:1105.4529.

[25] A. Neronov, D. V. Semikoz and C. Tchernin,
arXiv:1307.2158 [astro-ph.HE].

[26] S. Razzaque, Phys. Rev. D 88, $081302 \quad$ (2013) arXiv:1309.2756 [astro-ph.HE]].

[27] A. Levinson and E. Waxman, Phys. Rev. Lett. 87, 171101 (2001) hep-ph/0106102; C. Distefano, D. Guetta, E. Waxman and A. Levinson, Astrophys. J. 575, 378 (2002) astro-ph/0202200.

[28] M. M. Reynoso, G. E. Romero and H. R. Christiansen, Mon. Not. Roy. Astron. Soc. 387, 1745 (2008) arXiv:0801.2903 [astro-ph]].

[29] T. K. Gaisser, F. Halzen and T. Stanev, Phys. Rept. 258, 173 (1995) [Erratum-ibid. 271, 355 (1996)] arXiv:hep$\mathrm{ph} / 9410384$.

[30] T. K. Gaisser, arXiv:astro-ph/0501195

[31] T. K. Gaisser, J. Phys. Conf. Ser. 47, 15 (2006).

[32] W. D. Apel et al., Astropart. Phys. 47, 54 (2013).

[33] Though the damped pion decay chain does not yield flavor equipartition on Earth, any deviation from $\Phi_{0}^{\text {total }}$ falls in the range of uncertainty.

[34] Resonant $p \gamma$ interactions produce twice as many neutral pions as charged pions. Direct pion production via virtual meson exchange contributes about $20 \%$ to the total cross section, almost exclusively producing $\pi^{+}$. Hence, $p \gamma$ interactions produce roughly equal numbers of $\pi^{+}$and $\pi^{0}$.

[35] G. M. Frichter, T. K. Gaisser and T. Stanev, Phys. Rev. D 56, 3135 (1997) astro-ph/9704061.

[36] F. W. Stecker, Phys. Rev. Lett. 21, 1016 (1968).

[37] M. C. Chantell et al. [CASA-MIA Collaboration], Phys. Rev. Lett. 79, 1805 (1997) astro-ph/9705246.

[38] We thank Soeb Razzaque for pointing this out.

[39] A. Borione et al., Astrophys. J. 493, 175 (1998) astro$\mathrm{ph} / 9703063$.

[40] M. G. Aartsen et al. [IceCube Collaboration], Phys. Rev. D 87, no. 6, 062002 (2013) arXiv:1210.7992 [astroph.HE]].

[41] A. Gupta, S. Mathur, Y. Krongold, F. Nicastro and M. Galeazzi, Astrophys. J. 756, L8 (2012) arXiv:1205.5037 [astro-ph.HE]].

[42] F. C. Jones, A. Lukasiak, V. Ptuskin and W. Webber, astro-ph/0007293 V. S. Ptuskin, O. N. Strelnikova and L. G. Sveshnikova, Astropart. Phys. 31, 284 (2009). V. Ptuskin, Astropart. Phys. 39-40, 44 (2012).

[43] P. Lipari, arXiv:1308.2086 [astro-ph.HE].

[44] E. Waxman and J. N. Bahcall, Phys. Rev. D 59, 023002 (1999) arXiv:hep-ph/9807282. 